\title{
Editorial
}

\section{Ivermectin and COVID-19: Keeping Rigor in Times of Urgency}

\begin{abstract}
Carlos Chaccour, ${ }^{1,2,3 \star}$ Felix Hammann, ${ }^{4}$ Santiago Ramón-García, ${ }^{5,6}$ and N. Regina Rabinovich ${ }^{1,7}$
${ }^{1}$ ISGlobal, Hospital Clínic - Universitat de Barcelona, Barcelona, Spain; ${ }^{2}$ Ifakara Health Institute, Ifakara, United Republic of Tanzania; ${ }^{3} \mathrm{Facultad}$ de Medicina, Universidad de Navarra, Pamplona, Spain; ${ }^{4}$ Department of General Internal Medicine, Clinical Pharmacology and Toxicology, Inselspital, Bern University Hospital, University of Bern, Bern, Switzerland; ${ }^{5}$ Research \& Development Agency of Aragon (ARAID) Foundation, Zaragoza, Spain; ${ }^{6}$ Department of Microbiology, Mycobacterial Genetics Group, Preventive Medicine and Public Health, Faculty of Medicine, University of Zaragoza, Zaragoza, Spain; ${ }^{7}$ Harvard T.H. Chan School of Public Health, Boston, Massachusetts
\end{abstract}

Ivermectin is a widely used drug for the treatment and control of several neglected tropical diseases. ${ }^{1}$ The drug has an excellent safety profile, with more than 2.5 billion doses distributed in the last 30 years, and its potential to reduce malaria transmission by killing mosquitoes is under evaluation in several trials around the world. ${ }^{2}$ Ivermectin inhibits the in vitro replication of some positive, single-stranded RNA viruses, namely, dengue virus (DNV), ${ }^{3-5}$ Zika virus, ${ }^{4,6}$ yellow fever virus, ${ }^{7,8}$ and others. ${ }^{4,7,9}$

Caly et al. ${ }^{10}$ recently reported that ivermectin is a potent inhibitor of the severe acute respiratory syndrome coronavirus 2 (SARS-CoV-2) replication in vitro. Given the coronavirus disease19 (COVID-19) pandemic, this has understandably resonated widely in the global press. ${ }^{11}$

Caly et al. ${ }^{10}$ report a 5,000-fold reduction in SARS-CoV-2 RNA levels, compared with those in controls, after infected Vero/ hSLAM cells were incubated for 48 hours with $5 \mu \mathrm{M}$ ivermectin. The ivermectin $I \mathrm{I}_{50}$ for the virus was calculated at approximately $2.5 \mu \mathrm{M}$. These concentrations are the equivalent of 4,370 and $2,190 \mathrm{ng} / \mathrm{mL}$, respectively, notably 50 - to 100 -fold the peak concentration $\left(C_{\max }\right)$ achieved in plasma after the single dose of $200 \mu \mathrm{g} / \mathrm{kg}$ (14 mg in a 70-kg adult) commonly used for the control of onchocerchiasis. ${ }^{12}$ Pharmacokinetic studies in healthy volunteers have suggested that single doses up to $120 \mathrm{mg}$ of ivermectin can be safe and well tolerated. ${ }^{13}$ However, even with this dose, which is 10-fold greater than those approved by the US Food and Drug Administration, the $C_{\max }$ values reported were 250 ng/mL, ${ }^{13}$ one order of magnitude lower than effective in vitro concentrations against SARS-CoV-2.

These findings may seem to discourage follow-up clinical trials with ivermectin. However, some in vivo effect may be possible even if efficacious in vitro concentrations are physiologically unattainable. A recent phase III clinical trial in dengue patients in Thailand, in which a once-daily dose of 400 $\mu \mathrm{g} / \mathrm{kg}$ for 3 days was found to be safe but did not produce any clinical benefit, ${ }^{14}$ showed a modest and indirect in vivo effect against DNV. ${ }^{14}$ Previous work by Wagstaff et al. ${ }^{5}$ reported inhibition at much higher in vitro concentrations $(25 \mu \mathrm{M})$ in DNVinfected Vero cells. Both pharmacokinetic considerations and the relatively long incubation period of DNV might explain the lack of clinical efficacy. Until we have a better understanding of ivermectin's antiviral mode of action and of appropriate in vitro systems for testing, we caution against using findings in Vero cells as more than a qualitative indicator of potential efficacy.

Very recently, preliminary findings on a potential effect of hydroxychloroquine combined with azithromycin against

*Address correspondence to Carlos Chaccour, ISGlobal, Hospital Clínic - Universitat de Barcelona, Rosello 132, $5^{\mathrm{a}} 2^{\mathrm{a}}$, Barcelona 08036, Spain. E-mail: carlos.chaccour@isglobal.org
SARS-CoV-2 were widely publicized, ${ }^{15}$ leading to a surge in demand and self-medication, which resulted in serious harm in some cases and a stock shortage that jeopardized drug availability for other critical conditions for which hydroxychloroquine or chloroquine is the standard of care, that is, vivax malaria, rheumatoid arthritis, and systemic lupus erythematosus. Efficacy claims for hydroxychloroquine against COVID-19 have been questioned in follow-up trials using similar dosing regimens, ${ }^{16,17}$ and we await results of randomized, controlled clinical trials exploring treatment efficacy.

We believe the recent findings regarding ivermectin warrant rapidly implemented controlled clinical trials to assess its efficacy against SARS-CoV-2. These trials may open a new field of research on the potential use of avermectin antiparasitic drugs, including compounds with an improved pharmacokinetic profile, as antivirals. ${ }^{18}$ However, because of the following points, extreme due diligence and regulatory review are needed before testing ivermectin in severe disease.

First, ivermectin, which targets glutamate-gated chlorine channels in invertebrates, may cross-target the GABA-gated chlorine channels present in the mammalian central nervous system (CNS) and cause neurotoxicity. ${ }^{19}$ This is normally prevented by an intact blood-brain barrier (BBB), but in patients with a hyperinflammatory state, endothelial permeability at the BBB may be increased and cause leaking of drugs into the CNS, potentially causing harm. ${ }^{20,21}$

Second, boosted antiretrovirals such as lopinavir/ritonavir and darunavir/cobicistat, which have been widely used against SARS-CoV-2 based on limited evidence, and a number of other drugs, are potent inhibitors of cytochrome $\mathrm{P}_{450} 3 \mathrm{~A} 4$, the main metabolic pathway for ivermectin. Concurrent use of these drugs will result in increased systemic exposure to ivermectin. Furthermore, ritonavir and cobicistat can readily inhibit one of the main efflux pumps in the BBB, P-glycoprotein, further favoring neurotoxicity. ${ }^{22,23}$ However, it is encouraging that a recent analysis of ivermectin-related neurotoxic adverse events reported to the WHO Program for International Drug Monitoring found only one case of 1,668 reports in which concomitant use of antivirals was associated with neurotoxicity. ${ }^{24}$

Third, as earlier, available evidence suggests that levels of ivermectin with meaningful activity against SARS-CoV-2 would not be achieved without extraordinary, potentially toxic increases in ivermectin dosing levels in humans. However, evidence from animal models showing up to 3-fold higher levels in pulmonary tissue than in plasma 1 week after oral dosing leaves the door open for further research, in particular for the treatment of respiratory viruses. ${ }^{25,26}$

The discovery of ivermectin's activity against SARS-CoV-2 gives reason for hope, but off-label and compassionate use requires careful risk-benefit considerations, ${ }^{27}$ especially in 
critically ill patients. A path to consider is evaluation first of impacts on virologic outcomes in uncomplicated, low-risk patients early in the course of the disease. Well-conducted clinical trials informed by robust pharmacokinetic models should be considered to validate the impact before the use of ivermectin to treat SARS-CoV-2 is implemented.

Received April 8, 2020. Accepted for publication April 10, 2020.

Published online April 16, 2020.

Authors' addresses: Carlos Chaccour, ISGlobal, Hospital Clínic Universitat de Barcelona, Barcelona, Spain, Ifakara Health Institute, Ifakara, United Republic of Tanzania, and Facultad de Medicina, Universidad de Navarra, Pamplona, Spain, E-mail: carlos.chaccour@ isglobal.org. Felix Hammann, Department of General Internal Medicine, Clinical Pharmacology and Toxicology, Inselspital, Bern University Hospital, University of Bern, Bern, Switzerland, E-mail: felix.hammann@insel.ch. Santiago Ramón-García, Research \& Development Agency of Aragon (ARAID) Foundation, Spain and Department of Microbiology, Mycobacterial Genetics Group, Preventive Medicine and Public Health, Faculty of Medicine, University of Zaragoza, Spain, E-mail: santiramon@unizar.es. N. Regina Rabinovich, ISGlobal, Hospital Clínic - Universitat de Barcelona, Barcelona, Spain and Harvard T.H. Chan School of Public Health, Boston, MA, E-mail: rrabinov@hsph.harvard.edu.

This is an open-access article distributed under the terms of the Creative Commons Attribution (CC-BY) License, which permits unrestricted use, distribution, and reproduction in any medium, provided the original author and source are credited.

\section{REFERENCES}

1. Omura S, Crump A, 2014. Ivermectin: panacea for resource-poor communities? Trends Parasitol 30: 445-455.

2. The Ivermectin Roadmappers, 2020. A roadmap for the development of ivermectin as a complementary malaria vector control tool. Am J Trop Med Hyg 102: 3-24.

3. Tay MY, Fraser JE, Chan WK, Moreland NJ, Rathore AP, Wang C Vasudevan SG, Jans DA, 2013. Nuclear localization of dengue virus (DENV) 1-4 non-structural protein 5; protection against all 4 DENV serotypes by the inhibitor Ivermectin. Antiviral Res 99: 301-306.

4. Yang SNY, Atkinson SC, Wang C, Lee A, Bogoyevitch MA, Borg NA, Jans DA, 2020. The broad spectrum antiviral ivermectin targets the host nuclear transport importin alpha/beta1 heterodimer. Antiviral Res 177: 104760.

5. Wagstaff KM, Sivakumaran H, Heaton SM, Harrich D, Jans DA, 2012. Ivermectin is a specific inhibitor of importin alpha/betamediated nuclear import able to inhibit replication of HIV-1 and dengue virus. Biochem J 443: 851-856.

6. Barrows NJ et al., 2016. A screen of FDA-approved drugs for inhibitors of Zika virus infection. Cell Host Microbe 20: 259-270.

7. Varghese FS, Kaukinen P, Glasker S, Bespalov M, Hanski L, Wennerberg K Kümmerer BM, Ahola T 2016. Discovery of berberine, abamectin and ivermectin as antivirals against chikungunya and other alphaviruses. Antiviral Res 126: 117-124.

8. Mastrangelo $\mathrm{E}$ et al., 2012. Ivermectin is a potent inhibitor of flavivirus replication specifically targeting NS3 helicase activity: new prospects for an old drug. J Antimicrob Chemother 67: 1884-1894.

9. Lee YJ, Lee C, 2016. Ivermectin inhibits porcine reproductive and respiratory syndrome virus in cultured porcine alveolar macrophages. Arch Virol 161: 257-268.

10. Caly L, Druce J, Catton M, Jans D, KM W, 2020. The FDAapproved Drug Ivermectin inhibits the replication of SARS-CoV-2 in vitro. Antiviral Res. Available at: https://www.sciencedirect.com/ science/article/pii/S0166354220302011.

11. Slisco A, 2020. Anti-parasite Drug Used since 1980s May Help Stop Coronavirus, New Study Says. Newsweek. April, 04.
Available at: https://www.newsweek.com/anti-parasite-drugused-since-1980s-may-help-stop-coronavirus-new-study-says1496083. Accessed April 5, 2020.

12. Chaccour C, Hammann F, Rabinovich NR, 2017. Ivermectin to reduce malaria transmission I. Pharmacokinetic and pharmacodynamic considerations regarding efficacy and safety. Malar J 16: 161.

13. Guzzo CA, Furtek Cl, Porras AG, Chen C, Tipping R, Clineschmidt CM, Sciberras DG, Hsieh JY, Lasseter KC, 2002. Safety, tolerability, and pharmacokinetics of escalating high doses of ivermectin in healthy adult subjects. J Clin Pharmacol 42: 1122-1133.

14. Yamasmith E et al., 2018. Efficacy and Safety of /vermectin against Dengue Infection: A Phase III, Randomized, Double-blind, Placebo-controlled Trial. He 34th Annual Meeting the Royal College of Physicians of Thailand- 'Internal Medicine and One Health': Chonburi, Thailand. Registry. Available at: https:// clinicaltrials.gov/ct2/show/NCT02045069. Accessed Aplri 5, 2020.

15. Gautret $P$ et al., 2020. Hydroxychloroquine and azithromycin as a treatment of COVID-19: results of an open-label non-randomized clinical trial. Int $J$ Antimicrob Agents. Available at: https://www.sciencedirect.com/science/article/pii/ S0924857920300996.

16. Song S, Zhang D, Qian Z, Li T, Shen Y, Hongzh L, 2020. A Pilot Study of Hydroxychloroquine in Treatment of Patients with Common Coronavirus Disease-19 (COVID-19). Available at: http://subject.med.wanfangdata.com.cn/UpLoad/Files/202003/ 43f8625d4dc74e42bbcf24795de1c77c.pdf. Accessed April 5, 2020. Journal of Zhejiang University, 3.

17. Molina JM, Delaugerre C, Goff JL, Mela-Lima B, Ponscarme D, Goldwirt L, de Castro N, 2020 No evidence of rapid antiviral clearance or clinical benefit with the combination of hydroxychloroquine and azithromycin in patients with severe COVID-19 infection. Méd Mal Infect 9: S0399-077X(20)30085-8.

18. Scherr N, Pluschke G, Thompson CJ, Ramon-Garcia S, 2015. Selamectin is the avermectin with the best potential for buruli ulcer treatment. PLoS Negl Trop Dis 9: e0003996.

19. Menez C, Sutra JF, Prichard R, Lespine A, 2012. Relative neurotoxicity of ivermectin and moxidectin in Mdr1ab (-/-) mice and effects on mammalian GABA(A) channel activity. PLoS Negl Trop Dis 6: e1883.

20. Varatharaj A, Galea I, 2017. The blood-brain barrier in systemic inflammation. Brain Behav Immun 60: 1-12.

21. Poyiadji N, Shahin G, Noujaim D, Stone M, Patel S, Griffith B, 2020. COVID-19-associated acute hemorrhagic necrotizing encephalopathy: CT and MRI features. Radiology. Available at: https://pubs.rsna.org/doi/pdf/10.1148/radiol.2020201187.

22. Lepist El, Phan TK, Roy A, Tong L, Maclennan K, Murray B, Ray AS, 2012. Cobicistat boosts the intestinal absorption of transport substrates, including HIV protease inhibitors and GS-7340, in vitro. Antimicrob Agents Chemother 56: 5409-5413.

23. Drewe J, Gutmann H, Fricker G, Torok M, Beglinger C, Huwyler J, 1999. HIV protease inhibitor ritonavir: a more potent inhibitor of P-glycoprotein than the cyclosporine analog SDZ PSC 833. Biochem Pharmacol 57: 1147-1152.

24. Chandler RE, 2018. Serious neurological adverse events after ivermectin-do they occur beyond the indication of onchocerciasis? Am J Trop Med Hyg 98: 382-388.

25. Lespine A, Alvinerie M, Sutra JF, Pors I, Chartier C, 2005. Influence of the route of administration on efficacy and tissue distribution of ivermectin in goat. Vet Parasitol 128: 251-260.

26. Chiu SH, Lu AY, 1989. Metabolism and tissue residues. Campbell WC, ed. Ivermectin and Abamectin. New York, NY: SpringerVerlag, 131-143.

27. Kalil AC, 2020. Treating COVID-19-off-label drug use, compassionate use, and randomized clinical trials during pandemics. JAMA. Available at: https://jamanetwork.com/journals/jama/ fullarticle/2763802. 